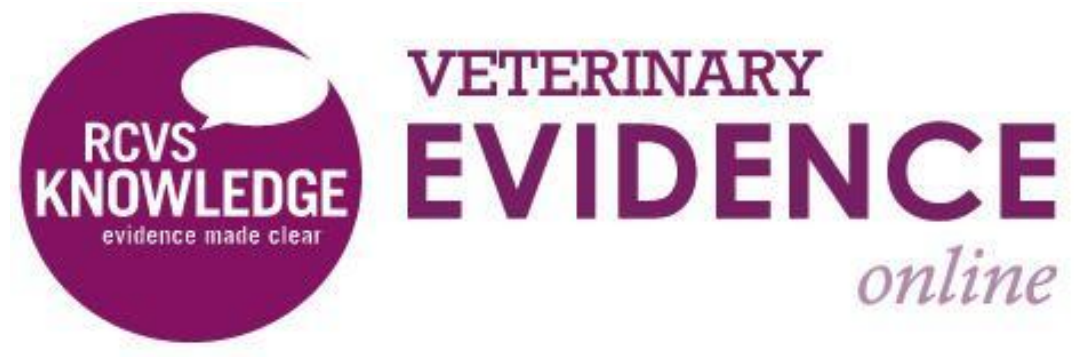

\title{
In Dogs With a European Adder Bite, Does the Use of Antivenom With Supportive Treatment Compared to Supportive Treatment Alone Improve Time to Recovery?
}

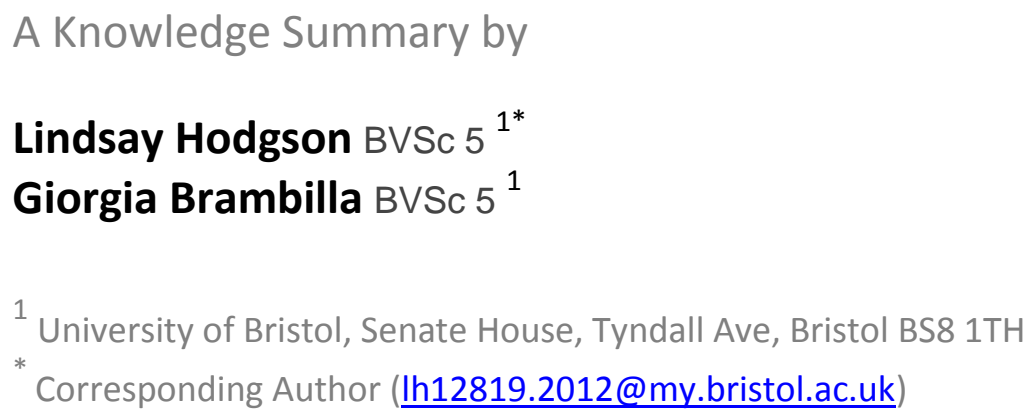

ISSN: 2396-9776

Published: 06 Nov 2017

in: Vol 2, Issue 4

DOI: http://dx.doi.org/10.18849/ve.v2i4.114

Reviewed by: Jackie Belle (RVN, CertSAN, CertFN, CertVNES, DipCABT, DipESM, Dip, BR, DipEnDv, PTLLS, BSc, MSc) Kit Sturgess (MA, VetMB, PhD, CertVR, DSAM, CertVC, MRCVS) and Nicola Bates (BSC MSc MA) 
KNOWLEDGE SUMMARY

\section{Clinical bottom line}

The current literature does not offer convincing evidence for the positive effect of antivenom on time to recovery in dogs envenomated by the European adder. It appears that the use of antivenom in addition to supportive treatment may positively affect local swelling if given within 24 hours of the bite, but the evidence is low quality and further studies are required before a more definitive answer can be reached.

\section{Question}

In [dogs with European adder bites] does the use of [antivenom and supportive treatment] compared to [supportive treatment only] [improve time to recovery]?

\section{Clinical Scenario}

A dog has been brought into your practice with a suspected European adder bite. You do not stock antivenom in the practice and you are aware that it can be difficult to access a source. You wish to know if there is any evidence that giving antivenom will improve the dog's recovery time, compared to supportive treatment alone.

\section{The Evidence}

The quality of the evidence available to answer the PICO question is limited by the lack of studies directly comparing the addition of antivenom to supportive treatment alone. At the time of writing no studies were found for the use of antivenom to treat European adder envenomation of dogs with time to recovery used as an outcome. Current studies are limited by bias; the decision to use antivenom is often made on severity of clinical signs, so that only the most severely affected dogs receive it Different sized dogs will also be affected with varying degrees of severity, with some dogs presenting asymptomatically. In addition, different amounts of venom may be injected with some bites injecting no venom (Sutton et al, 2011) so every dog is not subjected to the same amount of venom. These limitations influence the conclusions that can be drawn about the effect of antivenom on time to recovery.

In conclusion, a convincing body of evidence to influence the clinician's decision whether or not to use antivenom in the dog, in addition to supportive treatment, does not currently exist. Other considerations include difficulty in sourcing antivenom (Sutton et al, 2011), so if clinicians decide its use is warranted, they should begin locating a source as soon as possible. Adverse reactions to antivenom have also been reported and a study conducted on 54 dogs found that $7 \%$ of the patients developed at least one side effect after administration of $F(a b) 2$ antivenom. The reactions reported in this paper included facial swelling unrelated to the snake bite, profound panting and non-productive cough (Lund et al, 2013); anaphylaxis (Turkovic et al, 2015) has also been reported. At the time of writing, the authors were unable to find any reports of death in dogs directly caused by antivenom administration and Lund et al (2013) state that adverse effects appear to be "relatively mild and self-limiting". As with any drug, the side effects should be considered as part of the decision making process about whether or not to use antivenom. 


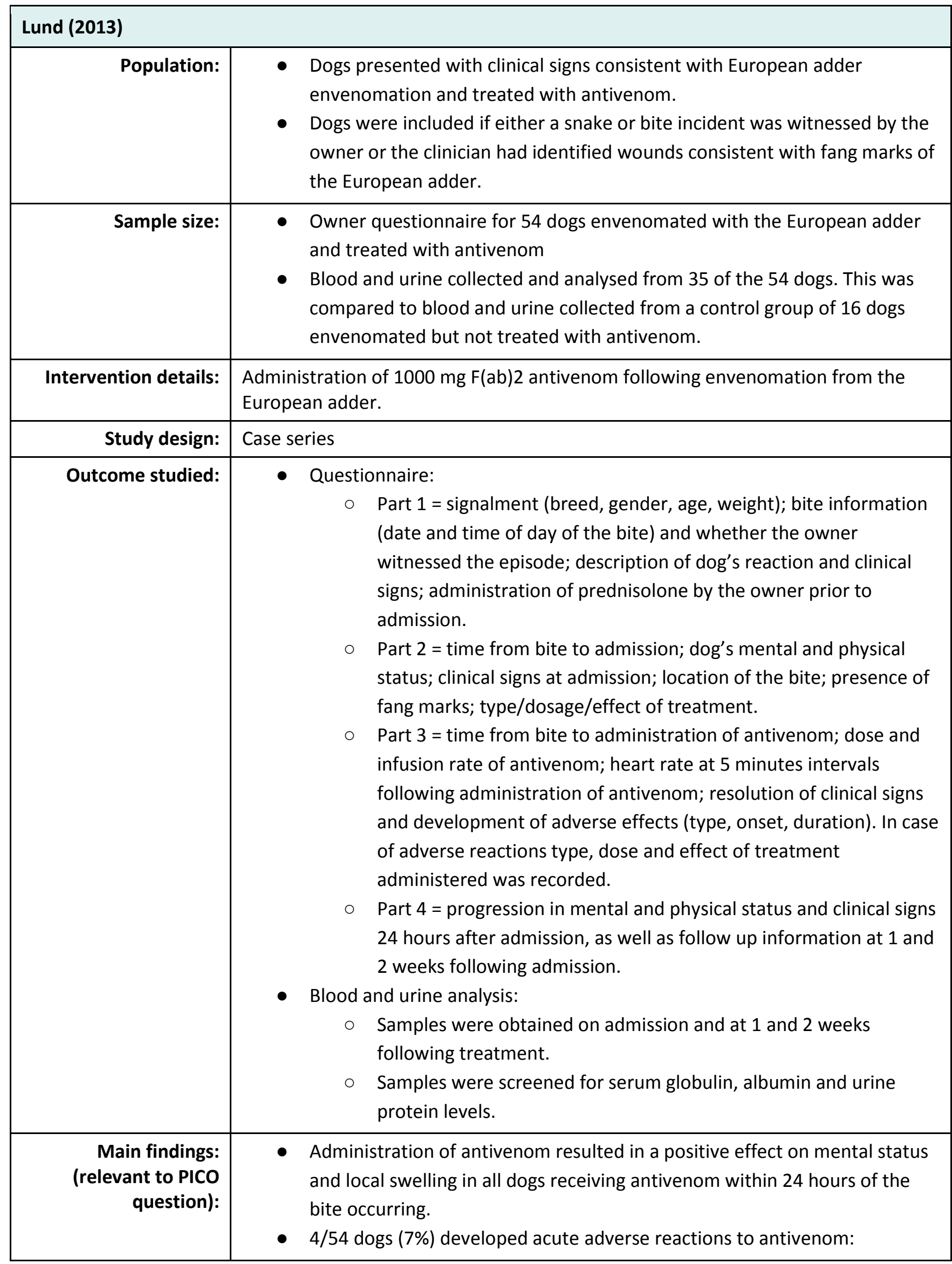




\begin{tabular}{|c|c|}
\hline & $\begin{array}{l}\text { o } 2 \text { dogs developed facial swelling } \\
\text { o } 1 \text { dog developed non-productive cough } \\
\text { o } 1 \text { dog developed sudden, profound panting } \\
\text { - Dogs treated with antivenom had a lower percentage of proteinuria after } 2 \\
\text { weeks compared to dogs not treated with antivenom }(P=0.03) \text {. } \\
\text { - No differences in serum albumin or globulin were observed between the } 2 \\
\text { groups. } \\
\text { - } 2 / 54 \text { dogs treated with antivenom died: } \\
\circ 1 \text { due to multiple organ failure after } 4 \text { days } \\
\circ 1 \text { was euthanased after } 5 \text { days due to kidney failure }\end{array}$ \\
\hline Limitations: & $\begin{array}{l}\text { Decision to use antivenom was made by different vets on a subjective } \\
\text { basis leading to a non-standardised intervention protocol. As a result, the } \\
\text { group of dogs receiving antivenom could have been more severely } \\
\text { affected compared to the control group of dogs. } \\
\text { - Multiple observers throughout the study and no standardised method for } \\
\text { assessment of mental status or local swelling were reported, leading to } \\
\text { lack of objectivity in improvements seen with antivenom use. } \\
\text { - Small sample size. } \\
\text { - Study group and control group were not randomly allocated. } \\
\text { Control group was composed of animals that could not receive antivenom } \\
\text { due to cost, lack of availability or deemed unnecessary by the clinician in } \\
\text { charge. As a consequence, the control group may include dogs with less } \\
\text { severe presentations. }\end{array}$ \\
\hline
\end{tabular}

\begin{tabular}{|c|c|}
\hline \multicolumn{2}{|l|}{ Sutton (2011) } \\
\hline Population: & $\begin{array}{l}\text { Dogs in the UK reported to the Veterinary Poisons Information Service } \\
\text { (VPIS) between September } 1985 \text { and December 2010, with European adder } \\
\text { bites. } \\
\text { Questionnaires requesting details of clinical effects, onset and duration of } \\
\text { effects, treatments given and clinical outcome are sent to around } 55 \% \text { of } \\
\text { enquirers, and patient records were only included if this follow up was } \\
\text { complete. }\end{array}$ \\
\hline Sample size: & $\begin{array}{l}985 \text { enquiries with follow up information from } 422 \text { cases and outcomes reported } \\
\text { in } 411 \text { cases. }\end{array}$ \\
\hline Intervention details: & $\begin{array}{l}\text { - IVFT used in } 241 \text { dogs ( } 57.1 \% \text { ). } \\
\text { - Antivenom used in } 236 \text { dogs ( } 55.9 \%) \text {. } \\
\text { - Supportive treatment included: } \\
\circ \text { Glucocorticoids used in } 216 \text { dogs. } \\
\circ \text { Antibacterial agents used in } 235 \text { dogs. } \\
\circ \text { Antihistamines used in } 84 \text { dogs. } \\
\circ \text { Combinations of supportive treatments given to individual dogs } \\
\text { were not reported. }\end{array}$ \\
\hline
\end{tabular}




\begin{tabular}{|c|c|}
\hline Study design: & Case series \\
\hline Outcome studied: & $\begin{array}{l}\text { - Age of dog, weight, sex, breed, month and time of bite and postcode of } \\
\text { reporting practice. } \\
\text { - Clinical signs reported, onset and duration of clinical signs. } \\
\text { - Time to response to antivenom. } \\
\text { - Duration of oedema. } \\
\text { - Death. }\end{array}$ \\
\hline $\begin{array}{r}\text { Main findings: } \\
\text { (relevant to PICO } \\
\text { question): }\end{array}$ & $\begin{array}{l}\text { Duration of oedema was reported as } 46.8 \text { hours in dogs that received } \\
\text { antivenom ( } n=39 \text { ) and } 94.1 \text { hours in those that did not receive antivenom } \\
\text { ( } n=52 \text { )(significance unknown - Sutton et al acknowledge that more data is } \\
\text { required to calculate significance due to the small number of cases where } \\
\text { this outcome was reported). } \\
\text { - Response to antivenom reported in } 15 \text { dogs; average response time was } \\
74.3 \text { minutes (range } 20 \text { minutes to four hours). Method of assessment was } \\
\text { not stated. } \\
\text { - Death occurred in } 7 \text { dogs of } 236 \text { receiving antivenom (3\%) (unknown if } \\
\text { euthanased or died). } \\
\text { Death occurred in } 9 \text { dogs of } 186 \text { not receiving antivenom (4.8\%) (unknown } \\
\text { if euthanased or died). } \\
\text { Of the } 189 \text { vets that had completed the free comments area of the follow } \\
\text { up questionnaire, } 33 \text { vets commented that antivenom gave a good clinical } \\
\text { response, } 18 \text { vets commented that antivenom response time was quick, } 11 \\
\text { vets commented that antivenom visibly improved oedema and one vet } \\
\text { commented that improvement was faster than without antivenom. Three } \\
\text { vets noted that antivenom appeared to have no effect, two vets } \\
\text { commented that antivenom made oedema worse and anaphylaxis was } \\
\text { reported in one dog following antivenom administration. } \\
\text { Authors of study note that no studies exist on the use of antihistamines for } \\
\text { envenomation. As venom causes local histamine release, this may be a } \\
\text { future area to study. }\end{array}$ \\
\hline Limitations: & $\begin{array}{l}\text { - Limited evidentiary value as retrospective review of records. } \\
\text { - No controls used. } \\
\text { - } \text { treatments, so unknown which treatment had effect on clinical signs and } \\
\text { - } \text { time to recovery. } \\
\text { - Dutcomes were not reported in all dogs, reducing the sample size. } \\
\text { - Limited follow up information given and quality of response varied widely. } \\
\text { - No statistical analysis of results given, so unknown if outcomes are } \\
\text { significant, although author notes that "antivenom administration did not }\end{array}$ \\
\hline
\end{tabular}




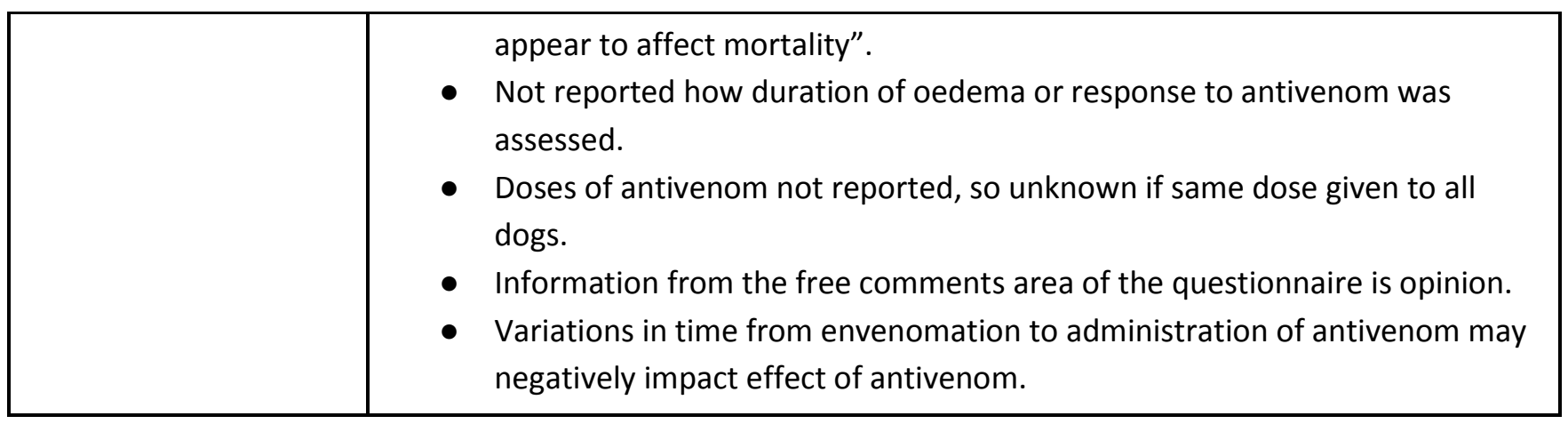

Turkovic (2015)

\begin{tabular}{|c|c|}
\hline Population: & $\begin{array}{l}\text { Dogs in Germany bitten by the European adder, presented to the Small Animal } \\
\text { Clinic at Ludwig-Maximilians University, Munich, between 1st January } 2008 \text { and } \\
\text { 31st August 2014. }\end{array}$ \\
\hline Sample size: & 15 \\
\hline Intervention details: & 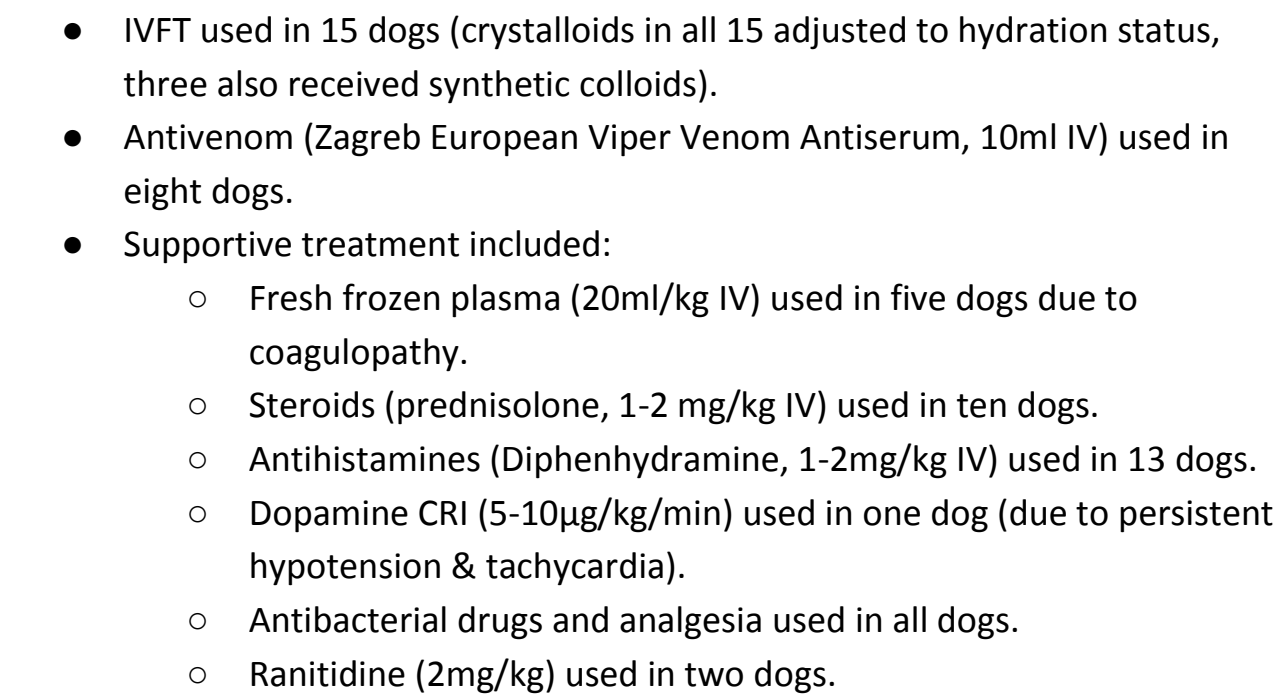 \\
\hline
\end{tabular}




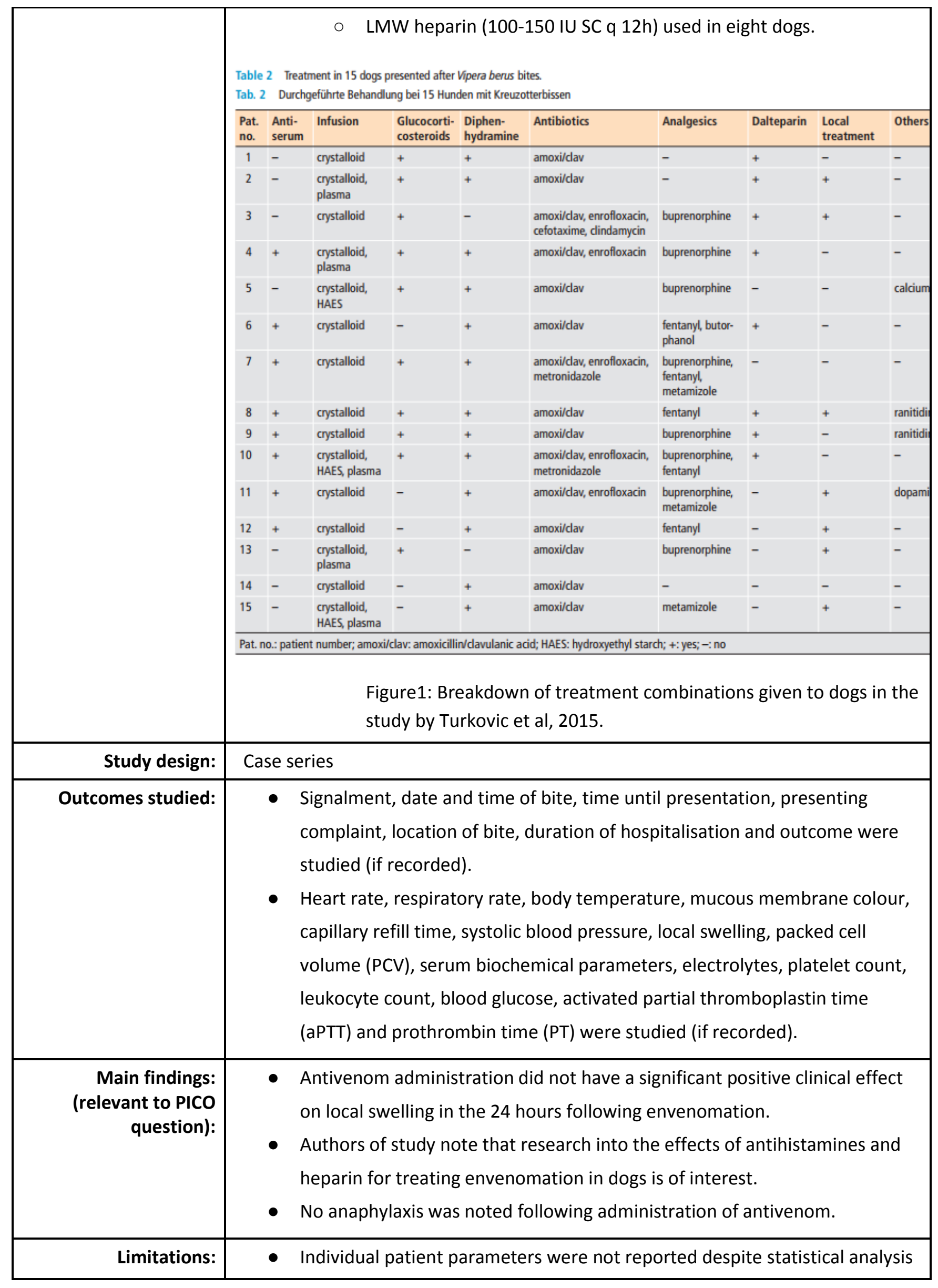




\begin{tabular}{|l|l|}
\hline being carried out. Data was incomplete and so correlations between \\
treatments and individual changes could not be made or analysed. \\
- Treatment and outcome monitoring protocols were not standardised \\
making it difficult to compare effects. \\
- Duration of hospitalisation was recorded for the dogs that did and did not \\
receive antivenom but results were not analysed for significance. \\
- No controls were used and so it is not possible to demonstrate if antivenom \\
affected outcome. \\
- Small study size. \\
- Rethod of measuring local swelling not stated.
\end{tabular}

\section{Appraisal, application and reflection}

The literature search performed by the authors found three papers which partially addressed the PICO question.

The study by Lund et al (2013) reports an improvement in local swelling and mental status in animals that received antivenom within 24 hours of the European adder bite. The group of dogs receiving antivenom also showed decreased proteinuria levels two weeks after the bite. These findings could be considered of interest when answering the PICO question; the study, however, presents some significant limitations which affect the quality of the evidence produced. The dogs recruited for the study had not been randomly assigned to the antivenom or the control group. The choice to administer antivenom was based on the clinician's subjective assessment of the severity of the clinical signs, the financial situation of the owner and the availability of antivenom. This could have led to the introduction of bias in patient selection. Moreover, the study was not blinded and the subjective improvement noted by the clinicians in swelling and mental status of dogs receiving antivenom cannot be regarded as good quality evidence for the PICO question.

In the Sutton et al (2011) study of cases reported to the VPIS, it was found that in dogs receiving antivenom, oedema lasted an average of 46.8 hours, compared to dogs that did not receive antivenom, where oedema lasted an average of 94.1 hours. This was the most significant finding to the PICO question in this paper but is limited by several factors. Duration of generalised oedema was not reported in all dogs, decreasing the sample size, and objective method of oedema measurement was not given. Statistical analysis of the findings was not reported, so it is not known if the findings are significant. Death occurred in $3 \%$ of dogs receiving antivenom and $4.8 \%$ of dogs not receiving antivenom, which appears similar, but unfortunately no conclusions can be drawn due to the lack of statistical analysis. The study looked at broad risk factors for envenomation and mortality and the scope of treatment, rather than evaluating the effect of any individual treatment, and this was a common theme among the evidence found.

Turkovic et al (2015) found that antivenom administration did not significantly clinically affect local swelling 24 hours after envenomation, which appears to contrast to the finding from the Lund paper (2013). This paper was another case series studying risk factors, treatments and outcomes, with a very small study size and data relevant to the PICO question was incomplete. Duration of hospitalisation was recorded for dogs receiving and not receiving antivenom but the results were unfortunately not analysed for significance. The use of heparin and antihistamines in treating envenomation were highlighted as future areas of research.

The majority of the studies found by the authors examine risk factors for envenomation, adverse effects of antivenom administration and common treatment choices. Furthermore, most studies were retrospective, considered envenomation by other species or studied the effects of other elements of the treatment regime, such as glucocorticoids. There is wide variation in the combinations of supportive treatment given in the literature; combinations of intravenous fluid therapy (IVFT) with crystalloids, colloids or blood products, analgesia, antibacterial agents, glucocorticoids, antihistamines and heparin (Sutton et al, 2011, Turkovic et al, 2015 and Lund et al, 2013). This lack of a standardised treatment protocol makes direct comparison of the effect of antivenom more difficult to assess. These variations affect the analysis of the impact of antivenom 
on time to recovery. The best study design to answer this PICO question would directly compare the outcomes when the use of antivenom is the only variable imposed by the study authors.

The PICO question could be best answered by a prospective, randomised, double-blinded controlled trial, comparing the effects of the addition of antivenom to supportive treatment alone. This could be considered as a research prospect; although a lack of antivenom licensed in dogs and the need to envenomate dogs would render such a study unlikely to pass ethics approval. The relatively small number of dogs envenomated each year in the UK could potentially render such a study financially unviable for antivenom manufacturers. Objective judgement of the clinical effects of antivenom, such as reduction of oedema, also generates difficulties. As such, the proposed clinical trial using ViperaVet may be of interest in revisiting this clinical question in the future; although at the time of writing the study was postponed (VPIS, 2017).

\section{Methodology Section}

\begin{tabular}{|c|c|}
\hline \multicolumn{2}{|l|}{ Search Strategy } \\
\hline $\begin{array}{r}\text { Databases searched and dates } \\
\text { covered: }\end{array}$ & $\begin{array}{l}\text { CAB Abstracts on OVID Platform } 1973 \text { - Week } 72017 \\
\text { Thomson Reuters Web of Science 1900-2017 } \\
\text { PubMed (any date) }\end{array}$ \\
\hline Search terms: & $\begin{array}{l}\text { 1. Dog* OR cani* OR bitch* OR pup* } \\
\text { 2. 'Viper* berus' OR 'European adder*' OR 'European viper*' OR } \\
\text { adder } \\
\text { 3. Antiven* OR antiser* OR anti-ven* OR anti-ser* } \\
\text { 4. } 1 \text { and } 2 \\
\text { 5. } 1 \text { and } 2 \text { and } 3 \\
\text { Please note the terms "adder bite*" and "envenomation" were also } \\
\text { included in the search strategy, but yielded fewer results than the } \\
\text { terms used above and yielded no additional papers. }\end{array}$ \\
\hline Dates searches performed: & $23 / 03 / 2017$ \\
\hline
\end{tabular}

Exclusion / Inclusion Criteria

\begin{tabular}{|c|c|}
\hline Exclusion: & $\begin{array}{l}\text { - Reviews of available treatments } \\
\text { - } \text { Expert opinion } \\
\text { - } \text { Letters } \\
\text { - } \text { Book chapters } \\
\text { - Papers studying envenomation by species other than the } \\
\text { - } \text { European adder or envenomation of humans } \\
\text { - } \text { translated) }\end{array}$ \\
\hline Inclusion: & $\begin{array}{l}\text { Studies regarding the use of antivenom for treatment of } \\
\text { envenomation of dogs by the European adder, Vipera berus. }\end{array}$ \\
\hline
\end{tabular}




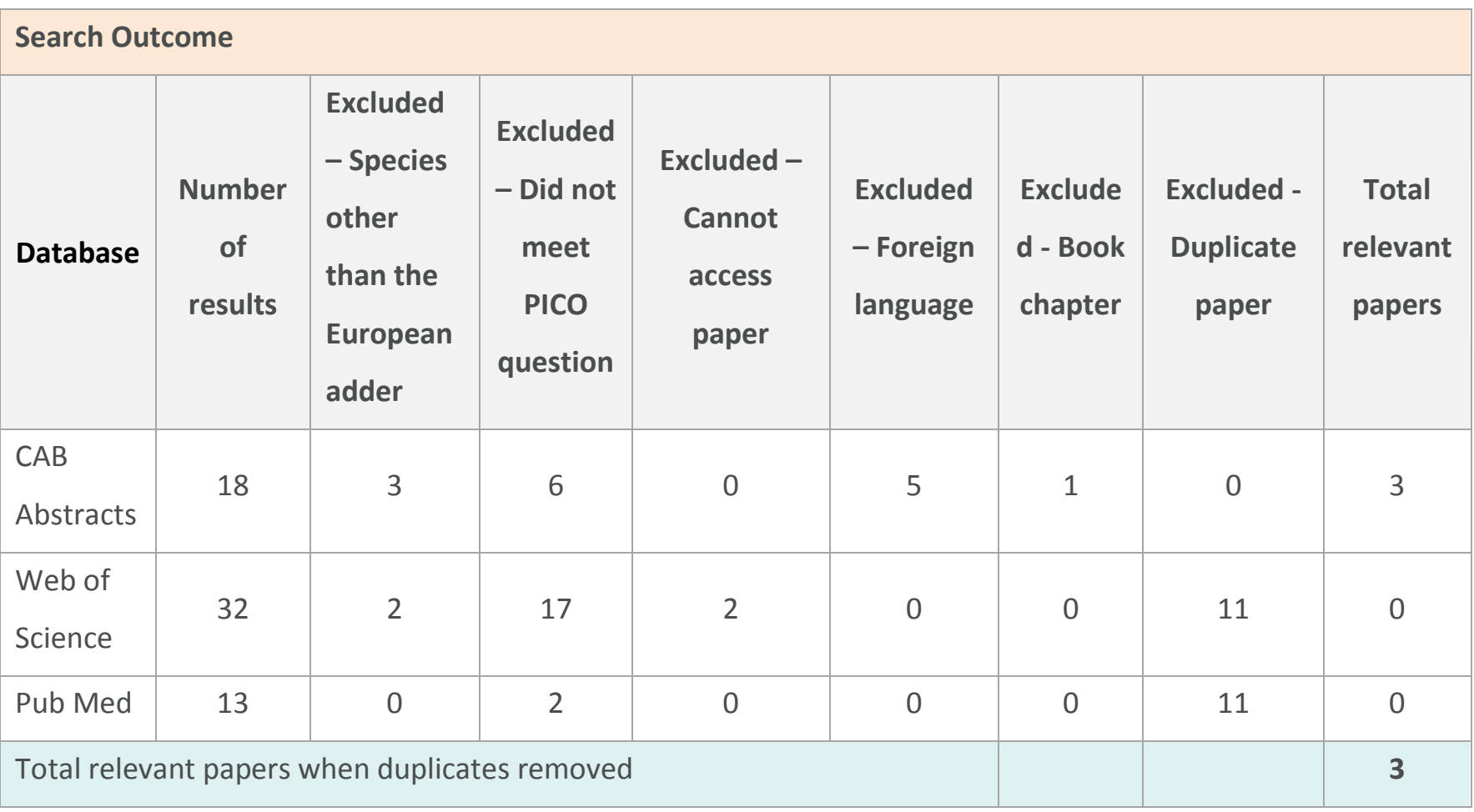

\section{CONFLICT OF INTEREST}

The authors declare no conflicts of interest.

The authors wish to thank Clare Boulton for her assistance with the literature search and Emma Place for her assistance with locating papers.

\section{REFERENCES}

1. Lund, H., Kristiansen, V., Eggertsdóttir, A. et al . (2013). Adverse reactions to equine-derived F(ab')2antivenin in 54 dogs envenomated by Vipera berus berus. Journal of Veterinary Emergency and Critical Care. 23 (5), 532-537. 2013. 1111/vec.12099

2. Sutton, N., Bates, N. and Campbell, A. (2011). Canine adder bites in the UK: a retrospective study of cases reported to the Veterinary Poisons Information Service. Veterinary Record. 169 (23), 607. 2011. http://dx.doi.org/10.1136/vr.d4695

3. Turkovic, V., Teichmann, S. and Doerfelt, R. (2015). European Adder bites in dogs in southern Germany. A retrospective study over a 6.5-year period. Tieraerztliche Praxis Ausgabe Kleintiere Heimtiere . 43 (4), 221-230. 2015. https://doi.org/10.15654/TPK-140364

4. Veterinary Poisons Information Service. (2017). Clinical trial for veterinary adder antivenom invitation to take part. Available: https://vpisglobal.com/adder-antivenom/ Last accessed 26th July 2017. 


\section{EVIDENCE \\ online}

\section{Intellectual Property Rights}

Authors of Knowledge Summaries submitted to RCVS Knowledge for publication will retain copyright in their work, and will be required to grant to RCVS Knowledge a non-exclusive license of the rights of copyright in the materials including but not limited to the right to publish, re-publish, transmit, sell, distribute and otherwise use the materials in all languages and all media throughout the world, and to license or permit others to do so.

\section{Disclaimer}

Knowledge Summaries are a peer-reviewed article type which aims to answer a clinical question based on the best available current evidence. It does not override the responsibility

of the practitioner. Informed decisions should be made by considering such factors as individual clinical expertise and judgement along with patient's circumstances and owners' values. Knowledge Summaries are a resource to help inform and any opinions expressed within the Knowledge Summaries are the author's own and do not necessarily reflect the view of the RCVS Knowledge.

Veterinary Evidence and EBVM Network are RCVS Knowledge initiatives. For more information please contact us at editor@veterinaryevidence.org

RCVS Knowledge is the independent charity associated with the Royal College of Veterinary Surgeons (RCVS). Our ambition is to become a global intermediary for evidence based veterinary knowledge by providing access to information that is of immediate value to practicing veterinary professionals and directly contributes to evidence based clinical decision-making.

www.veterinaryevidence.org

RCVS Knowledge is a registered Charity No. 230886. Registered as a Company limited by guarantee in England and Wales No. 598443.

Registered Office:

Belgravia House

62-64 Horseferry Road

London SW1P 2AF 